\title{
Autonomous Underwater Vehicle to Inspect Hydroelectric Dams
}

\author{
Edson Cavalcanti Neto \\ Federal Institute of Technology of Ceará \\ Av. Treze de Maio, 2081 \\ Benfica, Zip-code: 60040-531 \\ Guilherme C. Holanda \\ Federal Institute of Technology of Ceará \\ Av. Treze de Maio, 2081 \\ Benfica, Zip-code: 60040-531
}

\author{
Rejane C. Sá \\ Federal Institute of Technology of Ceará \\ Av. Treze de Maio, 2081 \\ Benfica, Zip-code: 60040-531 \\ Francisco A. X. da Mota \\ Federal Institute of Technology of Ceará \\ Av. Treze de Maio, 2081 \\ Benfica, Zip-code: 60040-531
}

\author{
Antonio T. Varela \\ Federal Institute of Technology of Ceará \\ Av. Treze de Maio, 2081 \\ Benfica, Zip-code: 60040-531
}

André L. C. Araújo

Federal Institute of Technology of Ceará

Av. Treze de Maio, 2081

Benfica, Zip-code: 60040-531
Ítalo J. Loiola

Universidade de Fortaleza

Washington Soares Av. 1321, B1. J-30

Edson Queiroz, Zip-code: 60811-905

\author{
Rogério Oliveira \\ Federal Institute of Technology of Ceará \\ Av. Treze de Maio, 2081 \\ Benfica, Zip-code: 60040-531
}

\author{
Auzuir R. de Alexandria \\ Federal Institute of Technology of Ceará \\ Av. Treze de Maio, 2081 \\ Benfica, Zip-code: 60040-531
}

\author{
Victor Hugo C. de Albuquerque \\ Universidade de Fortaleza \\ Washington Soares Av. 1321, B1. J-30 \\ Edson Queiroz, Zip-code: 60811-905
}

\section{ABSTRACT}

Driven by the rising demand for underwater operations in the fields of dam structure monitoring, ecosystems of reservoir lakes from Hydropower Plants (HPP) and mining and oil, underwater robotics is increasing rapidly. The increase in exploration, prospecting, monitoring and security in lakes, rivers and sea, both in commercial applications such as scientific applications, has led large companies and research centers to invest in the development of underwater vehicles. The purpose of this work is to develop and evaluate the performance of a dedicated expert system for an Autonomous Underwater Vehicle (AUV) to inspect hydroelectric dams, focusing efforts on mechatronic project based on dimensioning structural elements and machinery and elaborating the sensory part, which includes navigation sensors and sensors of environment conditions, as well as its vision system to detect and measure cracks on hydroelectric dams. The integration of sensors in an intelligent platform provides a satisfactory control of the vehicle, allowing the movement of the submarine on the three spatial axes. Because of the satisfactory fast response of the sensors, it is possible to determine the acceleration and inclination besides his attitude in relation to the trajectory instantaneously taken, and geometry and depth of the cracks. This vehicle will be able to
\end{abstract}

monitor the physical integrity of dams, making acquisition and storage of environment parameter such as temperature, dissolved oxygen, $\mathrm{pH}$ and conductivity as well as document images of the biota from reservoir lakes HPP, with minimized cost, high availability and low dependence on a skilled workforce to operate it.

\section{General Terms:}

AUV, Dams, inspection, navigation, Computer Vision

\section{Keywords:}

Autonomous Underwater Vehicle, Computational Vision, Dams, Underwater Robotics

\section{INTRODUCTION}

Unmanned Underwater Vehicles (UUVs) are mobile robots used to perform a wide range of activities in aquatic environments and used in some military, industrial (oil exploration and related activities) and in scientific areas such as marine biology [1].

The Remotely Operated Vehicles (ROVs) and Autonomous Underwater Vehicles (AUVs) are the two main subgroups of UUVs [19]. 
The first is characterized by receiving energy and exchange information with the control panel on the surface via an umbilical cord. From the control panel the operator can plan tasks or use a joystick to directly maneuver the vehicle, features which are absent in AUVs as they do not suffer the intervention of a human operator during the mission and also have no umbilical cord. The power supply is loaded onto the vehicle, as well as the central processing unit. Due to the lack of cable, autonomous vehicles have greater freedom of movement and its use is increasing because of advances in processors and means of energy storage, allowing greater autonomy to these vehicles.

In Brazil there are few studies on the field of underwater robotics. Dominguez [11] did a study on modeling and developed a program for dynamic simulation of underwater vehicles. Cunha [10] proposed an adaptive control system for tracking the trajectory. Hsu et al. [13] presented a procedure for identifying the dynamic model of the thrusters. Barros and Soares [3] presented a proposal for a low cost vehicle that can operate as ROV or AUV. Souza e Maruyama [18] investigated different control techniques for dynamic positioning.

Worldwide, there is a large number of published works. There are several areas of research in the general context of underwater robotics, such as modeling the interaction between fluid and structure [16], modeling of the actuators [7], control techniques for the vehicle [2], simulation environments [9] and vehicle design [14]. There are currently 110 hydroelectric plants in Brazil this number includes the small, medium and large. Conducting an assessment of physical condition of each dam is a task that requires time, money and skilled workers. Considering that the Brazilian energy matrix has $74 \%$ of its power generated by hydroelectric plants, the guarantee that the dams of these reservoirs are in good condition ensures the efficient production of energy for the whole population. An inspection task performed by a team of divers can take about a month to the end, depending on the size of the reservoir. With the inclusion of an autonomous underwater vehicle this task would take considerably less time. The use of an unmanned and autonomous vehicle for the maintenance of the dam provides safety and effectiveness for the activity.

Currently, the integrity evaluation of submerged structures is performed with the aid of piezometers, level references, surface landmarks, devices for containment and flow downstream measurement of the landfill. Although such devices resent valuable arguments for the maintenance of submerged structures, they do not allow inferences about the existence or characteristics of fractures and the proper localization of them. This assessment procedure is carried out by divers, who face the difficulty of water turbidity, which reduces visibility, interfering on an accurate evaluation of the structure and increasing the risk of diving.

Other important elements on the study for optimizing the operation and maintenance of HEP, such as monitoring of acidity, silting, control of shellfish, fish fauna, macrophytes and water quality, require additional mechanisms used with no association with the studies of maintenance. This increases the cost and takes the opportunity to multi-criteria analysis of cause and effect, essential for making strategic decisions.

Although research in the field of underwater vehicles is large, after a thorough review of the literature, as viewed previously, few studies were found dealing with the application of the AUV for inspection of dams.

This paper presents a project developed at the Federal Institute of Education, Science and Technology of Ceará (IFCE/Brazil) in partnership with the Hydroelectric Company of So Francisco (CHESF/Brazil), which aims on design, implementation and testing of an autonomous underwater vehicle (AUV) with capacity of autonomous locomotion, to perform monitoring of the physical structures of dams and capture information about the local biota based on mechatronic project using dimensioning structural elements and machinery and elaborating the sensory part, which includes navigation sensors and sensors of conditions, as well as its computational vision system to detect and measure cracks on hydroelectric dams. This paper is organized as follows: Section 2 presents an overview of the physical structure of the vehicle. Details of the electronic systems, instrumentation and hardware systems are shown on section 3 The description of the Robot Vision module is presented on Section 4 The experiments performed and results are discussed and analyzed in Section 5 Finally, conclusions and future proposals are fulfilled in Section 6

\section{PHYSICAL STRUCTURE}

The AUV developed in this study was designed and built following the paradigms of Mechatronics. According to Borenstein [8] a mechatronic system is not only a combination of electrical and mechanical systems and is more than just a control system, it is a complete integration between all of them. For those reasons this project has been supported by students and engineers from different areas (Mechanics, Robotics, Control, Telecommunications, Computer Science and Electronics) divided into the following steps:

(1) Mechanical Systems: CAD /CAE have been employed to design the prototype. The project took regard to various requirements, such as environmental conditions, the appropriate position of sensors on the platform, the position of the control system, speed and maximum depth desired, etc. Then the prototype was built and assembled using CAD designed in the first step.

(2) Electronic systems: Some sensors were evaluated and the most suitable were acquired. In addition, a computer has been selected to run the control and navigation programs of the vehicle in an autonomous way. Along with the computer, some tasks are performed by distributed systems that are micro controlled and connected via an appropriate communication bus.

(3) Information Technology: Simultaneously with the previous phases, strategies for autonomous navigation and controls of trajectory were analyzed and studied.

\subsection{Composition}

In Figure 1 the physical structure of the AUV developed it?s shown. It consists on three parts: (a) a tube at the top center, where the sensors and electronic systems responsible for processing and control of the vehicle are located; (b) two tubes in lower base, where the floating, submersion and emergence systems are; (c) two propellers, fixed above the lower tubes, responsible for the rotation and translation of the submarine.

It assumes an AUV form as described in block diagram of the Figure 2 The AUV is composed of multiple peripherals, including acceleration sensors, guidance and pressure to power the robot navigation system and sensors for analysis of water quality. The sensors and actuators are connected to different microcontroller processing units, interconnected to each other forming a distributed architecture, in which no single system is in hierarchical level higher than any other, making the system robust, reliable, flexible, extensible and fault tolerant. The failure of one subsystem does not necessarily imply the failure of the global system. The navigation and control system is based on a PC. 


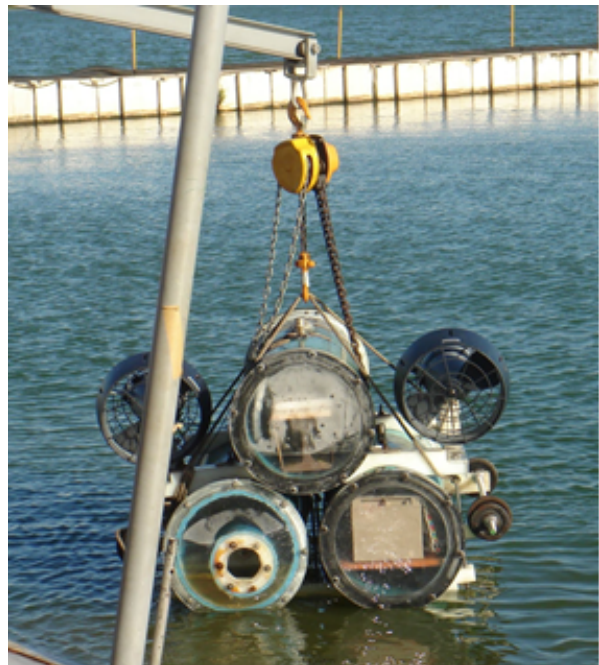

Fig. 1. Physical structure of the submarine.
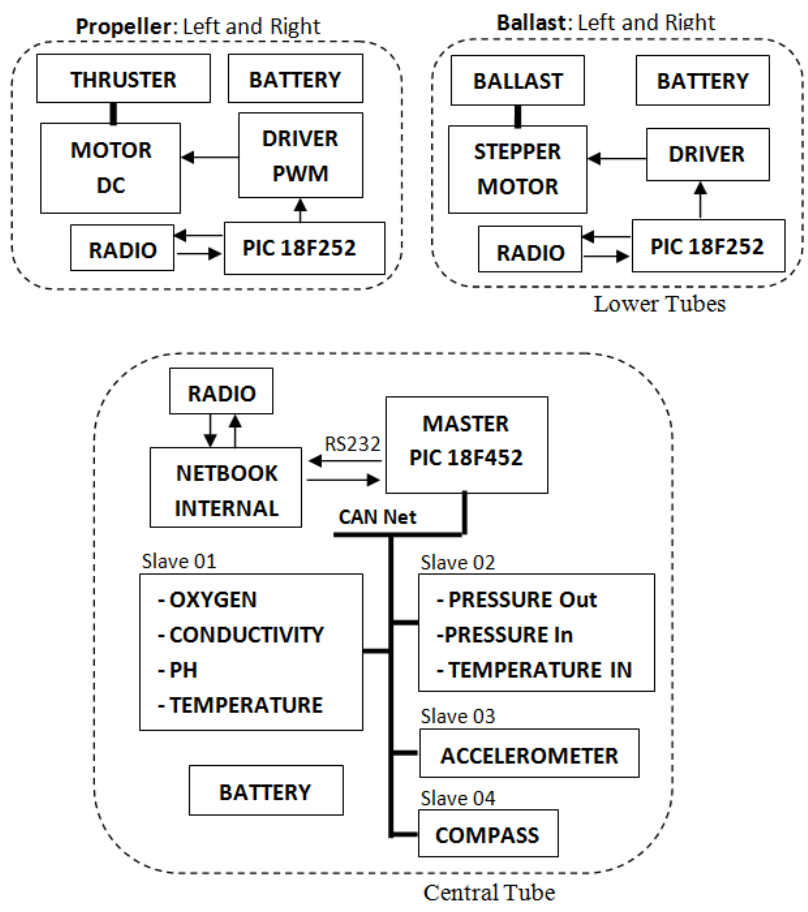

Fig. 2. Block Diagram of the AUV.

A system of radio frequency communication was an incorporated between PC and microcontroller processing units of the ballast system and drivers, because they us different lines. The units of instrumentation that are on the same tube as the PC, are linked by means of a CAN (Control Area Network). The vehicle also has an image acquisition system for inspection of the dam walls. In Figure 2 is presented an illustration of the block diagram of the AUV.

\subsection{Floatation}

The submarine has an initial volume of approximately $70.685 \mathrm{dm}$ on each of the side bases and $35.342 \mathrm{dm}$ in the central body; therefore, to keep in balance, it can support a mass of up to approximately $175 \mathrm{~kg}$, so the thrust is slightly larger than the weight which makes it float.

Initially the submarine has to be on the water surface; in this case the thrust is greater than the weight to pop it always floating on the surface, which does not generate disorder, because it?s possible to compensate the balance with buoys or weights.

\subsection{Flotation control}

To control its floatation, emergence and submergence, will be used two pistons coupled individually on the side bases. Today, some submarines use a pod system to carry out this task, for this project a plunger-ballast system was chosen because, in addition to saving energy to perform the tasks of immersion and emersion, it does not affect the capture of images that is made to verify the structure of the dams.

According to the laws of conservation of mass, the mass of a body cannot vary, but it is possible to change its volume. This variation is what makes the submarine performs submersion and emersion. A slight variation in the difference between mass and volume changes the balance of the body, causing it to sink, balance or come to the surface in the liquid. The entire length of each side of the bases is used to control this difference, they are now called, compression tubes.

Each of the compression tube consists of a plunger, a stepper motor, a rack, a rail and a support base for all these components as illustrated in Figure 3

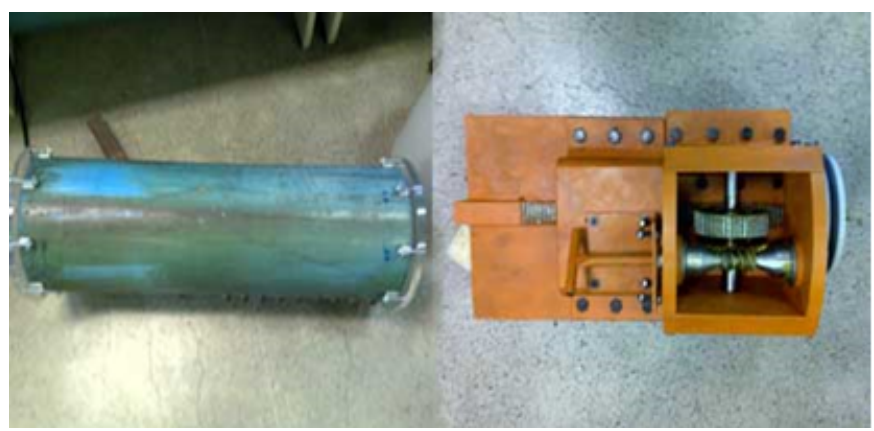

Fig. 3. Lower Tube and rack.

The engine makes the rack moves horizontally on the rail by pushing the plunger back and forth, according to the need of the submarine to stay submerged or emerged.

At the end of each tube there is an opening, through which the water fills the tube. When the piston is at its starting point (3/4 of total length), the thrust is equal to the weight and the submarine emerges in equilibrium. When the plunger is moved to occupy $2 / 4$ of the tube, the volume will decrease, because the rest is occupied by water, and the submarine sinks. By moving along the entire length of the tube, the volume increases and the submarine comes to the surface, remembering that to keep it in balance it?s necessary to move the tube to its starting point( $3 / 4$ of total length). 
Within each tube compression, there is also a system of plates (control, communication, RF and driver) and battery will be detailed in the following sections.

\subsection{Engine}

This is a delicate point of the project, because the engine indicates the maximum depth of efficient work of the submarine. The submersion depth of $10 \mathrm{~m}$ was adopted. With this data, the ideal engine was chosen.

According to the calculations, an engine with torque of $300 \mathrm{~kg}$ was regarded as optimal for successfully pushing the well-lubricated plunger to a depth of $10 \mathrm{~m}$ with security.

\subsection{Reduction}

The method used in the reduction is the "Crown and worm" due to the size and stability of the submarine, the engine should be in the same tube axis, so the force on the output shaft of the reduction should be applied to 90 of the input shaft. The pair ?crown and worm" is easily made, it gives great powers, has a lower cost, higher durability, transmits high torque with relatively small relationships, occupies small space when compared to other options and has the option of self-locking.

Despite many advantages, it also has disadvantages. For the case of the submarine, the ideal is a reduction of only $3: 1$, but reductions in the range of 10:1 to 2:1 it needs a large number of entries in the worm, and a very high helix angle, making its manufacture extremely complicated and expensive.

For this reduction with the advantages above it has been chosen the design with only one entry. Besides the fact that the "worm" has only a few entries, it was necessary to scale the helix angle to ensure the self-locking, so that the cosine of the friction angle must be greater than the cosine of the helix angle, so for the calculations of scaling, the reduction has a ratio of 14.5:1 and a helix angle of 20 .

The crown of this reduction could not be used to transfer motion to the rack because of its shape of coupled with the worm, so in the same axis it was placed a helical gear teeth to transmit the movement to the rack, so the gear box is divided into two parts, facilitating the scaling:

- Transfer of Power: The pair "crown and worm" that increase the engine torque to the value specified for the project.

-Transmission of Motion: The pair "Crown and rack" that uses the power gained from the "Crown and the worm" to move the plunger.

\subsection{Gasket}

The gasket of the submarine is made from aluminum parts properly machined with brackets for screws and o-rings, at the end of each tube of $150 \mathrm{~mm}$. The plunger, gasket is made by two o-rings that depending on the thickness can stand pressures from depths up to $100 \mathrm{~m}$.

To increase the gasket, facilitate the movement and lifetime of the plunger, silicone grease is used on the o-rings (better known as vacuum grease), because it is not soluble in water and prevents corrosion. In other words, lubrication is made with silicone grease using only alight film that, besides increasing the sealing will act as permanent lubricant, reducing friction.

\subsection{Propulsion}

For the horizontal motion of the submarine based thrusters, two drivers manufactured by Sea-Doo are used as illustrated in Figure 4.

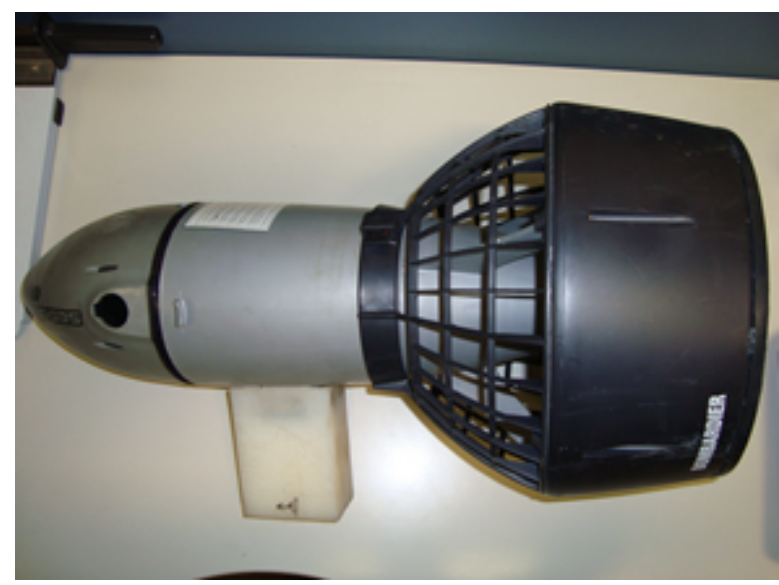

Fig. 4. Thrusters used.

These propellants are widely used by divers, capable of moving a weight of $150 \mathrm{~kg}$ approximately, which, according to the scaling of the submarine, are totally applicable to the task of displacement of the vehicle. Inside each propeller, there is a system of plates (control, communication and driver) and drums.

\section{EMBEDDED INSTRUMENTATION}

Sensors are devices that change the behavior when subjected to the action or form of energy. Many are assembled in the form of ICs (Integrated Circuit). They are used in many applications on electronics, especially in control and regulation systems, instrumentation, processing and signal generation or a physical quantity, which may directly or indirectly provide a sign indicating this greatness. Robots that work placed in real, static or dynamic environments have sensors that give them the ability to acquire information about the way they interact with the environment in which they operate and about their own internal state.

The submarine sensor system is composed of two parts: one concerning the navigation of the vehicle and another referring to the capture of environmental data. The integration of these sensors in an intelligent platform provides a satisfactory control of the vehicle, allowing the movement of the submarine in the three spatial axes.

\subsection{Navigation sensors}

Navigation is one of the most important aspects of underwater vehicles, to the extent that any use of a vehicle of this type largely depends on ability to trace a designated path, or know where it is when there is some important event. A great problem with navigation comes down to be able to determine, at every moment, the point in space where the vehicle is. For this purpose, we used two different sensors: acceleration and guidance.

\section{-Accelerometer}


Accelerometers are inertial sensors used in inertial navigation systems to determine the accelerations of the vehicle, in addition to use as inclinometer, essential to maintain the stability and linearity of the vehicle.

The accelerometer used: MEMS Freescale Semiconductor, MMA7260QT model shown in Figure 5 is an inertial sensor, with three axis, which has a selectable range from $+/-1.5 \mathrm{~g},+$ $/-2 \mathrm{~g},+/-4 \mathrm{~g}$ or $+/-6 \mathrm{~g}$ and operates within a temperature range from -20 to 85 .

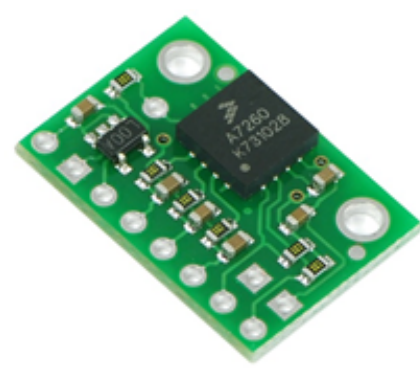

Fig. 5. Accelerometer used.

This sensor has a sensitive element, able to feel acceleration, and an interface ASIC, capable of transforming the information from the sensitive element to an analog signal, allowing it to capture. It should be noted that the detection of the signal direction is immediate, in any of the three axes, which helps to understand what the direction of movement, without the need for any calculations. The use of accelerometers deployed on electronic chips to make inertial systems is achievable for two reasons: first because it allows building a low-cost system, in addition, all the inertial system, using a microcontroller for processing the electrical signals sent by the accelerometer can be mounted in a single printed circuit board small.

\section{-Digital Compass}

The compass provides the direction in space that the vehicle is following. Figure 6 shows the electronic compass used, HMC6352 model, manufactured by Honeywell. The HMC6352 combines two magneto-resistive sensors in two axes, fully integrated, micro-processor and all the algorithms required to determine the orientation.

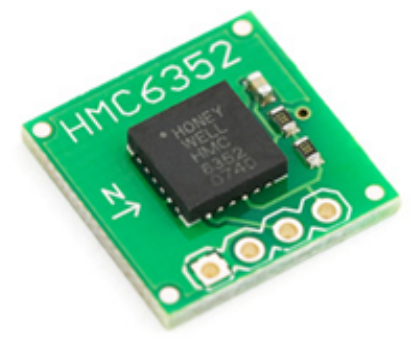

Fig. 6. Compass HMC-6352.

The module provides the steering angle, including temperature compensation algorithms and protection against magnetic parasite-fields. The signal of the compass is acquired through an I2C interface. Because the sensors used having a satisfactory response in a timely manner, it is possible to determine the acceleration and the inclination of the vehicle, and their attitude to the trajectory taken instantaneously.

\subsection{Environmental sensors}

The sensory part for the monitoring of environmental conditions of the dam consists of: $\mathrm{pH}$ sensor (EPC-70 model), temperature (SC-200), level of oxygenation (SO-400) and electrical conductivity (SC-100), all of them manufactured by Instrutherm.

The sensors are located outside the structure of the submarine, attached to the frame with fixed structures. The sensor cables enter the structure through an opening, which is properly sealed to prevent water from entering the internal structure.

All data from the sensors are captured by a communication network and stored in an embedded microcomputer. Subsequently, this data will be sent to a specialist who will make a study of the environmental conditions.

The main characteristics of the sensors used are summarized in Table 1.

\subsection{Internal Communication}

Autonomous vehicles are typically controlled from a control system that is composed of sensor units, actuators and processing center. Sensors and actuators can be connected to a single processing system forming a centralized architecture or related to different processing units interconnected to each other forming a distributed architecture.

The processing systems of modern robotic systems are usually distributed, which requires a shared memory or a data communication network between the processing units to ensure adequate time constraints for transmission of messages. A data communication network that has this ability to communicate in real time is a CAN (Control Area Network).

The CAN network is a multi-master serial bus for distributed control system that has as main features: prioritized bus access, reconfiguration flexibility, high reliability in noisy environments and a sophisticated and robust system of error detection.

Dealing specifically with the submarine, the information that travels over the CAN network is the navigation data: acceleration in three axes (x, y, and $\mathrm{z})$ and orientation of a digital compass, and sensor data of water quality, levels of oxygen, conductivity, water temperature and $\mathrm{pH}$ besides data from the internal temperature and internal and external pressure. The CAN network of the vehicle is formed by 5 nodes as shown in Figure 7

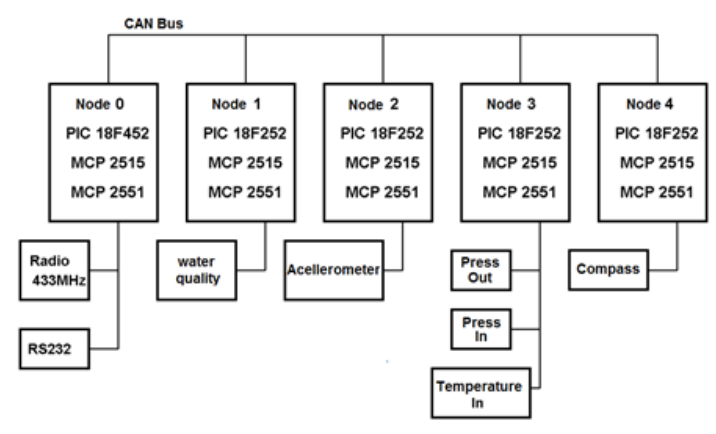

Fig. 7. Bus of CAN network. 
Table 1. Characteristics of installed sensors Digital Compass.

\begin{tabular}{|c|c|c|c|c|}
\hline Sensor & Manufacturer & Range & Precision & Signal \\
\hline Digital Compass & Honeywell/HMC-6532 & $20 \mathrm{e}$ & $0,5 \mathrm{deg}$ & $\mathrm{I} 2 \mathrm{C}$ \\
\hline Accelerometer & Freescale/MMA 6270QT & $1,5 \mathrm{~g}-6 \mathrm{~g}$ & $800 \mathrm{mV} / \mathrm{g}$ & $0,85 \mathrm{~V}-2,45 \mathrm{~V}$ \\
\hline External Pressure & NUOVA F./TPI-PRESS & $0-2$ bar & - & $4-20 \mathrm{~mA}$ \\
\hline Internal Pressure & Novus/NP-430D & $0-2$ bar & 0,002 bar & $4-20 \mathrm{~mA}$ \\
\hline Temperature In & National/LM-35 & $+2 \mathrm{C}-+150 \mathrm{C}$ & $0,5 \mathrm{C}$ & $10 \mathrm{mV} / \mathrm{C}$ \\
\hline Dissolved Oxygen & Instrutherm/SO-400 & $0-20.0 \mathrm{mg} / \mathrm{L}$ & $0,4 \mathrm{mg} / 1$ & RS232 \\
\hline $\mathrm{PH}$ & Instrutherm/EPC-70 & 0 a $14 \mathrm{pH}$ & - & RS232 \\
\hline Conductivity & Instrutherm/SC-100 & 0,2 a $2,000 \mathrm{mS}$ & (3\% e.c.) & RS232 \\
\hline Water Temp. & Instrutherm/ST-200 & 0 a $65 \mathrm{C}$ & $0,8 \mathrm{C}$ & RS232 \\
\hline
\end{tabular}

The submarine navigation computer is in direct communication with the "master" board or "Node 0", this board is responsible for receiving data from all sensors via the CAN network and sending command to activate the propellers and weights. The "nodes: $1,2,3$ and 4" perform the measurement in which the sensors are connected and send to the "Node 0" through the CAN network, as shown in Figure 7

Due to the small number of messages present in the AUV until then (less than 20), the standard CAN 2A is utilized and due to software implementation and use of codes already provided by the manufacturer of the CAN controller, the baud rate of the network is $125 \mathrm{Kbits} / \mathrm{s}$.

This system is located in the central tube, along with the electronics for sensing and control. Figure 8 shows a real image of the plates connected to the CAN network.

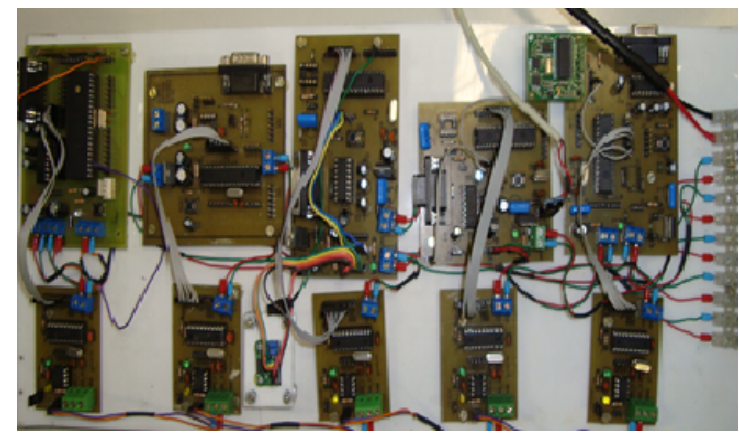

Fig. 8. CAN Network Platform.

\subsection{External Communication}

Distributed systems of the submarine must act together. That means that the elements: ballast, propellant and CAN network must work in synchronism to move the vehicle. For this combined work is necessary to send messages and commands to each part of the structure of the submarine. However, as these elements are independent and placed in different locations of the vehicle, there is the need for wireless communication.

When it comes to the underwater communication, there are serious problems, given that in theory radio waves do not propagate in water. However, after some testing a radio module was found, ER400TRS model, Transceiver manufactured by EasyRadio, which had a limit of wave propagation in water up to 2 meters. Such a feature of this radio made possible the integration of the systems: CAN network, ballast and thrusters.
In the literature there are several approaches to solving the problem of communication of mobile robots. The ability to form ad-hoc (self-forming, self-maintaining) networks from an assorted collection of platforms, ranging from simple sensors to unmanned autonomous vehicles to manned submersibles, would allow a richly interactive environment for data collection, surveillance, data distribution and collaborative planning and processing. Traditional existing architectures for distributed control of robotic and/or sensor systems have assumed a fixed, high bandwidth environment. This has enabled adaptive communications architectures to evolve, which supports powerful distributed applications such as distributed simulations, supercomputer based analysis of distributed databases, and so forth. While current network based distributed computing techniques exist, they are characterized by high bandwidth requirements. In this regard, these techniques are not suited to the underwater domain. The environment in which autonomous undersea systems must operate is fundamentally different due to the low bandwidth inherent to undersea communications systems. Benton [6], Benton [12] and Mupparapu [15] propose architectures for distributed control in Underwater Vehicles and ad hoc routing protocols.

This work will discuss only the internal communication between the propulsion systems, ballast and control system based on a modular RF (Radio Frequency).

3.4.1 Radio Mode. The ER400TRS consists on a low-power RF transceiver, a microcontroller and a voltage regulator, as shown in Figure 9 The microcontroller is responsible for the serial interface to the host system and RF transceiver functions. It also stores configuration data for the various operation modes of the transceiver module. The module has one output (Received Signal Strength Indicator - RSSI) that is used to measure the levels of the received signal.

The transceiver module operates at a $433 \mathrm{MHz}$ frequency of PanEuropean band and it?s fed by a $3.6 \mathrm{~V}$ source, transmitting input and output data serially. For better accuracy in the frequency of the synthesizer RF module, it is controlled by crystal. The receiver module has a high sensitivity, 105dBm@19.2 Kbps and the transmitter output power uses up to $10 \mathrm{~mW}$, and can be programmed by the user, as well as the operating frequency and data rate.

Figure 10 shows how the communication takes place between two radio modules and how the ER400TRS is connected to the microcontroller. Communication between the module and the microcontroller is carried out with two serial control signals, the first signing when the module is busy performing some internal task and the other for receiving data stored in the buffer module (Host Ready). The voltage levels used by radio module levels are TTL (Transistor Transistor Logic), while the netbook uses the standard serial RS232. To match the voltage levels its used a TTL/RS232 converter. 


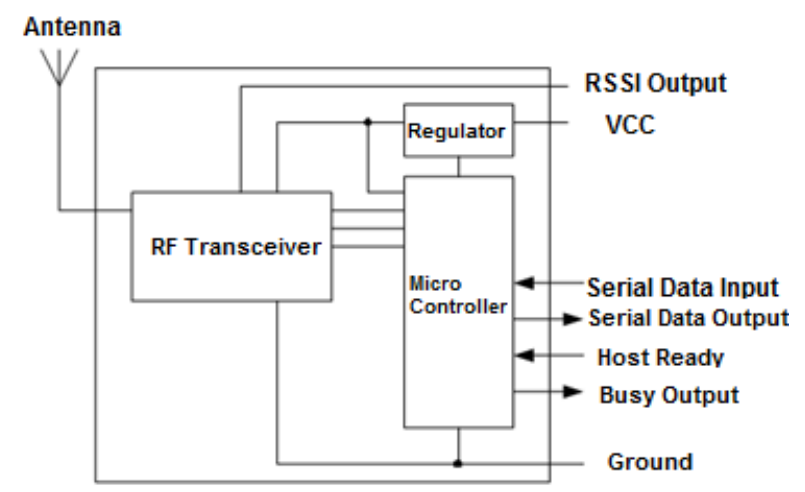

Fig. 9. Block diagram of the transceiver module.

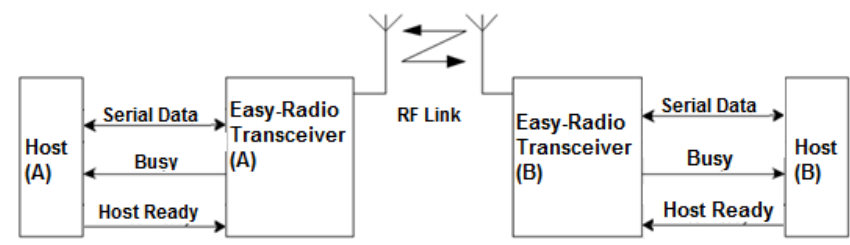

Fig. 10. Connecting the module to the host.

As shown in Figure 2 underwater wireless communication occurs between the two drivers, both internal ballast and netbooks, which also receives data from all sensors connected to the CAN network and performs the system control. The data sent by the netbook to the propeller is:

-Reference speed of the propellers;

- Constant control (Kp, Ti and Td);

-Request the current speed;

-Request the battery voltage;

The data sent by the netbook to the ballasts is:

-Depth reference;

- Constant control (Kp, Ti);

-Request the current position;

-Request the battery voltage;

\section{DESCRIPTION OF THE ROBOT VISION MODULE}

The computer vision robot module consists of sub-modules responsible for tasks connected with the inspection mission of dams or other submerged structures. These sub-modules are: image registration, crack detection, distance measurement, generation of mosaics and location of robot by images. For this, a stereo camera, model komiko, was installed in the robot cargo bay and linked to a netbook with this configuration, Windows XP. The stereo camera has two sets with optical CCD sensor. The images generated by the camera are recorded in the form of mpeg video along the mission. Upon returning from the mission, it is permitted to transfer the video file and your viewing in any video players, in which it is possible to activate or deactivate the crack detection system. This detection system allows automatic detection of cracks in the dam.
The distance measurement system uses images obtained from the stereo camera in order to carry out, through 3D vision algorithms, measuring the depth dimension. In this way, it is possible to measure the distance from the robot to the obstacles and, above all, distance measurement of the robot and the dam. The maintenance of the robot to a fixed distance of the structure allows for better image detection of cracks and the composition of the mosaics (feature where you can add several pictures to form a single more detailed). The mosaic generation system is responsible for the formation of a photographic map of the wall of the dam which facilitates the location of robot, registration and monitoring of location and size of detected cracks. Finally, a robot monitoring system is implemented by comparing the image of the wall from the live camera, with the photographic map obtained by the generation of mosaics.

\subsection{Detection and analysis of crack}

Crack detection module uses the images of the registration module interface images and simultaneously performs a treatment in the image to highlight the crack in the picture when exist. The technologies used for crack detection in images are the application of Adaptive threshold, the adaptation by medium and crack detection by the size of the area.

The application of Adaptive threshold consists of applying a mask $3 \times 3$ in the array of pixels to increase the points of interest. The adaptation by average consists of the calculation of the average value obtained from the pixels and from this average value is carried out a sweep in the array of pixels and is shown in a different color of the image to illustrate the location of the crack. The indication and decision of whether or not crack in the image is held from the calculation of the area of the crack in the image, namely having the value of the area the interface tells you whether or not there is crack on the image.

\subsection{The 3D vision for distance measurement}

The classic form of distance measurement is via sonars and radars lasers. However, the use of computer vision in stereo, based on two cameras is quite widespread in robotics. Recent points detection algorithms allow the development of different methods of stereo vision. Among these new algorithms is the SURF (Speeded up Robust Features Up) that can be used in conjunction with classic stereo vision algorithms, development of 3D computer vision systems.

Stereo imaging systems consist of effecting the simultaneous acquisition of two images of the same scene, in a manner similar to animals that have two eyes. With this system it is possible to rescue the depth information present in the acquired images.

In the literature there are works in which uses infrared sensor for the measurement of distance, but one of the drawbacks is the fact that the very narrow detection beam [5]. On the one hand it is an advantage because you can map more accurately the environment around, but on the other hand it cannot detect a little obstacle.

The algorithm SURF [4] includes key points detectors and descriptors. Surfing uses a special representation known as image in order to speed up the discovery process. Key points detection is performed from the construction of a representation in space-image scaling.

The scale-space representation is performed from the convolution of the image with a Gaussian kernel. This representation is a special type of multi-scale representation which covers a continuous scale parameter and saves the same spatial sampling for all scales. In this way, one can understand the scale-space of an image as a set of images of the same size as the original image [17]. 
The detection of characteristics within the SURF range uses the determinant of Hessian matrix. To find correspondences between key points of two images from their respective descriptors, it is necessary to find the best candidates to be their equivalents.

This is accomplished for the nearest neighbor point descriptor among all possible candidates. The minimum Euclidean distance between the descriptors. But not always the closest neighbour is the corresponding, therefore it is necessary to use the Euclidean distance with second nearest neighbour, being excluded matches that have the ratio of nearest neighbor and the second greater than a certain threshold, removing that large portion of false corresponding points. Also used the sign of the Laplacian, which distinguishes the brightness of points in dark backgrounds of the reverse situation, as shown in Figure 11

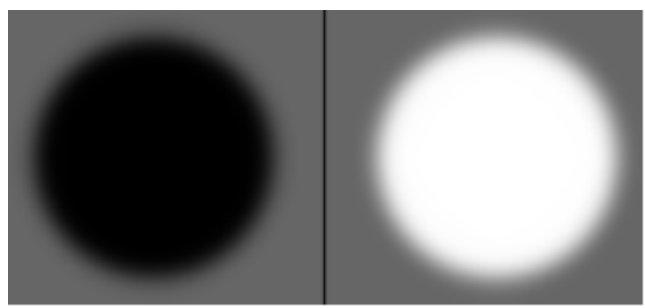

Fig. 11. Distinction brightness on points with dark backgrounds from the reverse situation Laplacian signal.

In the correspondence of points, compare only key points that have the same types of contrast. So, this little piece of information allows a faster matching phase, without reducing the performance of the descriptor. In short, if the contrast between two points of interest is different, the point is not considered a possible candidate corresponding point.

Below is a summary of the steps in the process of calculating depth:

(1) Capture: you get the images of the left and right cameras in RGB color scale of resolution $320 \times 240$ pixels. The two images must contain the object that you want to calculate the distance.

(2) Preprocessing: the images in the RGB scale are converted to grayscale, this process is useful in order to be a continuity between radiometric and pixels so that the images can serve as input to the algorithm SURF.

(3) Detection of image features: from the SURF algorithm key points are obtained both images with their respective descriptors.

(4) Matching key points: from the key points with their respective local descriptors, the SURF algorithm identifies corresponding points in the two images, which means identifying that point of the image right is corresponding to a given point of left image.

(5) Calculating depth: knowing the pairs of corresponding points, calculate the depth.

\subsection{Formation of mosaic}

A typical mobile robot is composed of the following subsystems: perception; location; navigation and control of movement. The perceptual system is responsible for the acquisition of environmental data that the robot operates. These data, in addition to being stored as part of the robot mission, are machined to be used in the generation of information concerning the location of robot in your environment, through its tracking system. Of possession of its location, the robot is able to generate a map of the environment that operates and compare your local position with a more global position on a map generated or delivered by its developer or user. The ability to navigate using the above map, supported on the mission that should play is also extremely necessary task and held by your navigation system. The latter tells which trajectory the motion control system must play.

The Mosaic can be defined as a conjunction of images of approximate scale, of a particular region, clipped and assembled, artistic or technically in such a way as to give the impression that such a set is a single image. Mosaic is the map described earlier and where by it the mobile robot can locate and navigate.

From the mosaic navigation is possible in the environment in which the robot is located. Using the Scale-invariant feature transform (SIFT), one can track points in images, even in real time, and perform the composition of mosaics.

The process of creating mosaics is accomplished by 4 steps: (i) capturing the images that constitute the mosaic, (ii) digital preprocessing of images, (iii) treating the image from a computer system to identify points of interest and similar images and (iv) creation of mosaic.

A mosaic visualization system is necessary, because for him be a join of multiple images is necessary to decrease the range, thus enabling the handling of images on the computer screen.

A recently used approach in obtaining critical points is the SIFT. Through it is possible to obtain automatically an image descriptors and use them to compare with other image descriptors. Thus, it is possible to calculate the offset and the guidance of critical points from one image to another, enabling the composition of the mosaics.

This mosaic will be used for detection of flaws or cracks in structures of a dam that will be obtained through the images acquired by a robot submarine. In addition to being used in the proper location of the robot. The navigation module, the topological map, provided by the user, of the mosaic obtained, the robot position on this map and the mission to be accomplished, defines the trajectory that the robot must perform.

During the assembling of a mosaic technique form, several difficulties may arise. And these can generate prejudice both in geometry as image preview (Mosaic) obtained. The main difficulties are: no coincidence of geometric features on the image and radiometric continuity not of pixels between the images.

For the use of robot vision system for localization, navigation and search for flaws in the structure of the dam, the mosaic composition becomes quite useful. The mosaic becomes, after the Union of numerous pictures obtained when travelled the whole dam, a real photographic map of the structure. This enables analysis and location of faults in the structure of the dam, both in an automatic way, how much visual form by operator. In addition, the robot can use mosaic to perform location estimation. To do this, again makes use of the and comparison of current image with mosaic built or under construction.

\subsection{SIFT Transformed}

SIFT is a robust method to extract and describe key points. The features extracted by this technique are invariant to translation, rotation, scaling, and partially invariant to changes in lighting and perspective [1]. Basically, the algorithm is performed in four steps: (i) detection of extremes: do a search on all scales and locations 
of images with differences of properties filters (DOG-Difference of Gaussian) for the identification of points of interest, rotation and scale invariant (ii) location of key points: where is an extreme, adjusts a comprehensive model to determine the exact location and scale. Key points are selected based on stability measures, (iii) definition of guidance: sets up an orientation for each key point using the gradients and image sites (iv) descriptor of the key points: the local gradient of each key point, based in the neighborhood.

These measures are transformed to a representation that is tolerant to significant levels of distortion and change in lighting. From obtaining key points of an image and another, these can be compared through little of Euclidean distance, and their corresponding. Thus, it is possible to trace points in images, even in real time, and issued the composition of mosaics.

\section{EXPERIMENTAL TESTS AND DISCUSSIONS}

After the physical structure and peripherals being properly treated, severe experiments will be conducted for calibration and testing of the sensory system, floatation control, navigation and inspection, in order to evaluate performance and validate the developed platform. Although some experimental results of navigation are presented (trajectory control and image processing) these issues will not be discussed in detail in this work because the necessary techniques for this are relatively complex and deserves a unique space to give a proper treatment to this problem.

\subsection{1st Step (gasket, immersion and emersion)}

The first step is to verify the existence of problems concerning the sealing in the submarine. For this, tests were performed in a swimming pool as shown in Figure 12

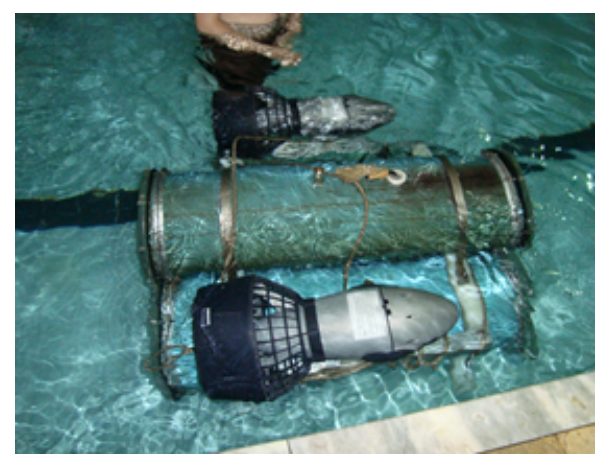

Fig. 12. Test in the pool.

During these tests, the emergence and submergence system was also observed, which processes were performed by the ballast located in the side tubes.

The mission, a term used to indicate the trajectory of the test, consisted in sending commands to the system of weights for immersion and emersion. Acting almost immediately, the vehicle moved satisfactorily, without making any change of inclination. Later on each test, the presence of leaks in the structure of the material was checked.

\subsection{2nd Step (sensors and communication)}

The second step is related to the acquisition of data from navigation sensors and sensors for the environment through the CAN net- work, in addition to activities already undertaken previously. At first, these data were used to identify the dynamic model of the robot that is not addressed in this work.

The implemented CAN network has provided a good position to evaluate that performance. It was possible to see the advantages that are obtained by using a distributed architecture such as: robustness, reliability, speed and expandability to new control points. As observed, the implementation of a CAN bus involves concepts of hardware, which is strongly dependent on the strategy of using the software and microcontroller.

So in the end of the second step, after some tests in a swimming pool, a test was performed in a foreign field, the dam Gavião, in possession of the Companhia de Gestão dos Recursos Hdricos (COGERH), illustrated in Figure 13

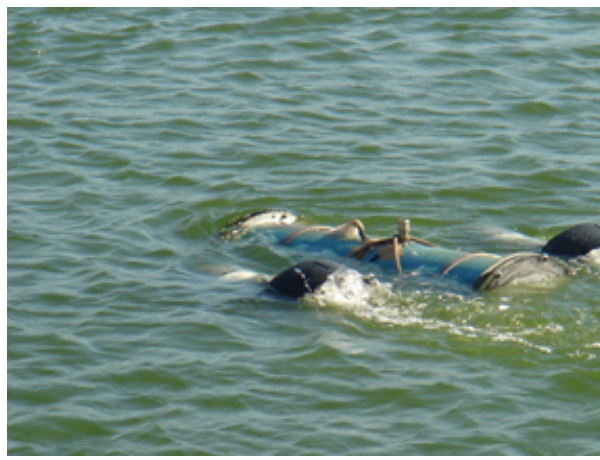

Fig. 13. Test at Gavião dam.

\subsection{3rd Step (navigation, control and validation)}

In this step, the screening tests of trajectory were performed based on data from the accelerometer and compass.

The control system must not only compensate the nonlinearities of the dynamics of the vehicle, but also the dynamics that were not modeled, or unstructured uncertainties, as well as external disturbances. These uncertainties include ocean currents, the hydrodynamics of the actuator system and the delays of navigation and control subsystems.

The goal stated in this step consists in some preliminary tests of trajectory control in the horizontal plane to evaluate vehicle performance and validate the developed platform. The study of control position was conducted with the use of linear PID control technique, due to the ease of implementation and tuning of controller parameters. The vehicle performed well following the planned trajectory, reaching the final desired position and orientation.

\subsection{4th Step (inspection)}

The first step for inspection module tests were using sintectic images to test the algorithm. The images that were used to test the algorthm is shown on Figure 14

The images were processed by the algorithm proposed and the final mosaic was evaluated. The result mosaic is shown on Figure 15

The informations about the results is shown on Table 2 To test the algorithm was used a total of 55 images and all the images were processed in $2 \mathrm{~ms}$. The signal/noise ratio was of $13 \mathrm{~dB}$ and the percentage of overlaping between the original iamge and the final image was of $93 \%$, in other words, the algorithm obtained a fast and accurate result. 


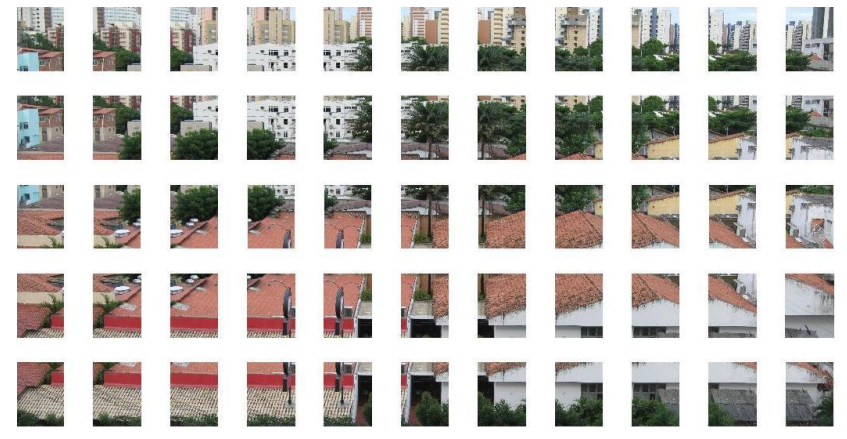

Fig. 14. Images to test the mosaic creation algorithm.

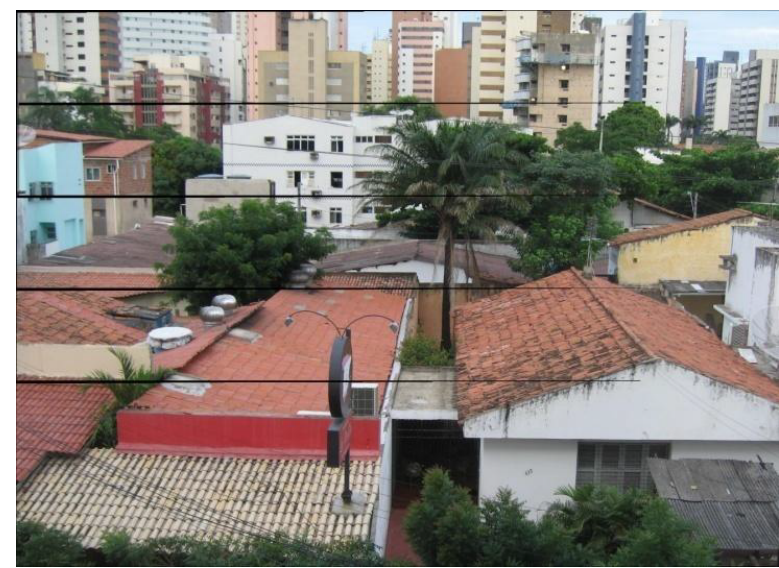

Fig. 15. Images to test the mosaic creation algorithm.

Table 2. Informations about the test

\begin{tabular}{|c|c|c|c|c|}
\hline & Quantity & Signal/Noise ratio & $\%$ of overlap & time \\
\hline Test & 55 images & $13 \mathrm{~dB}$ & 93 & $2 \mathrm{~ms}$ \\
\hline
\end{tabular}

The inspection module for detection of cracks uses images of the interface from registration module of images and simultaneously performs a treatment on the image to highlight the crack in the image, if any. The technologies used to detect cracks in images are the application of adaptive threshold, adjusting for average detection of crack by the size of the area. The application of adaptive threshold consists of applying a mask $3 \times 3$ in the matrix of pixels to increase the points of interest. The adaptation by average consists in calculating the average value obtained from the pixels and from this average, a scan is performed in the matrix of pixels and it is illustrated in a different color of the image to illustrate the location of the crack. The indication and decision the existence or not of crack in the image is performed by calculating the area of the crack in the image, that is, having the value of the area, the interface reports whether the image is broken. Initially, a test was performed to detect cracks in a pool as seen in Figure 16

Finally, a test to detect cracks from computational vision system, descript in the section 4, is performed in a video about an underwater inspection of a dam, shown in Figure 17

Figure 17 was generated from the grouping of different images (mosaic) fairly reliable in relation to the real. For this, some adjustments for better tracking and better generation of this mosaic were

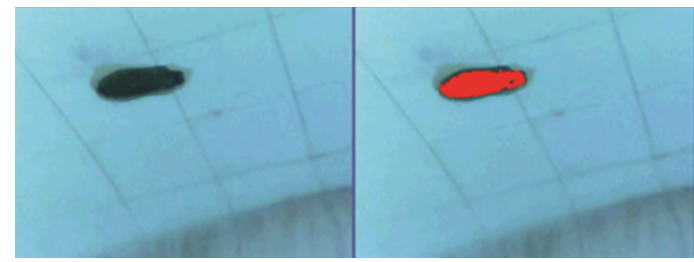

Fig. 16. Pool inspection.

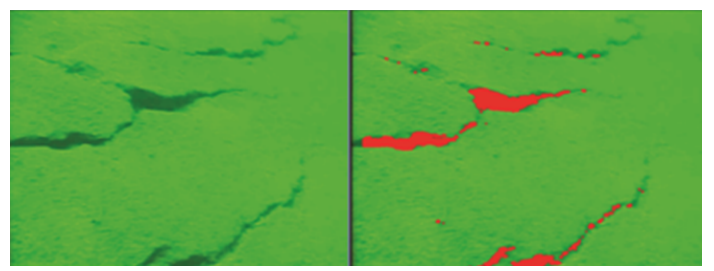

Fig. 17. Inspection to detect cracks from CHESF dam.

needed. In Figure 18 for forming printed images mosaic obtained from underwater video are presented.

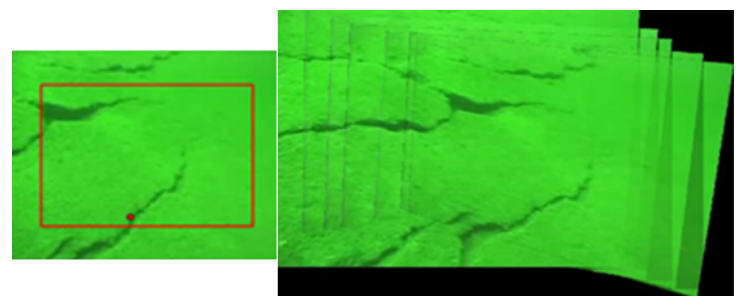

Fig. 18. Creating the mosaic.

The location of the robot in the mosaic uses the technology of obtaining SIFT critical points. From the critical points obtained from the mosaic and the current image, comparing the points of found images and correlated from the Euclidean distance is performed. After obtaining the correlated points is possible to identify the position of the current frame in the mosaic. This step is also important because the distance between the AUV and the obstacle is obtained through the computer vision. On Table 3 is shown the distance tests where five objects were used in different distances from the AUV. The percentage of error was low and the timo of processing was lower than $300 \mathrm{~ms}$.

\section{CONCLUSION AND FUTURE WORKS}

This work presents a mechatronic description of an AUV for inspection tasks in dams. The AUV developed is based on the synergistic integration of mechanical engineering, electronics and control.

The mechanical design and computational vision system of the mobile robot was performed using technologies of CAD/CAE in which the main features of the dam and its peripherals were considered. Then, some sensors were installed on the platform, one part of these sensors is used by the control system and navigation and other to the water quality analysis. Finally, the AUV was subjected to several tests in real environment in order to validate the vehicle. Experimental tests were successful and the preliminary results 
Table 3. Informations about the distance test

\begin{tabular}{|c|c|c|c|c|c|}
\hline & Real Distance $(\mathrm{mm})$ & Distance calculated by the algorithm & Number of interest points & Percentage of error(\%) & Time of processing (s) \\
\hline Object A & 400 & 401 & 15 & 0,25 & 0,142 \\
\hline Object B & 460 & 447 & 9 & 2,83 & 0,311 \\
\hline Object C & 330 & 310 & 5 & 6,06 & 0,132 \\
\hline Object E & 560 & 580 & 8 & 3,57 & 0,256 \\
\hline
\end{tabular}

showed the proper behavior of the vehicle to work on the proposed environment.

Combine the safety and efficiency to an activity is a challenge of any inspection project, the underwater robot developed integrates these two skills, with an immediate and achievable solution for monitoring the integrity of dams.

Perspectives for future works will focus on the dynamic modeling and advanced control techniques (predictive and adaptive) to provide better navigation performance of the vehicle. The aim is also include other navigation sensors such as sonar and gyroscope to improve the system location of the submarine.

\section{Acknowledgements}

This work has been supported by the Hydroelectric Company of San Francisco (CHESF/Brazil) and National Council for Scientific and Technological Development $(\mathrm{CNPq} / \mathrm{Brazil})$.

The authors thank the Federal Institute of Education, Science and Technology of Ceará (IFCE/Brazil) for the support, giving installations for the tests of the submarine and Company Water Resources Management (COGERH) for giving one of its testing facilities in the field.

\section{REFERENCES}

[1] P Angeli, Carla M Prado, Débora G Xisto, Pedro L Silva, Caroline P Pássaro, Hugo D Nakazato, Edna A LeickMaldonado, Milton A Martins, Patricia R M Rocco, and Iolanda F L C Tibério. Effects of chronic l-name treatment lung tissue mechanics, eosinophilic and extracellular matrix responses induced by chronic pulmonary inflammation. American Journal of Physiology - Lung Cellular and Molecular Physiology, 294:197-205, 2008.

[2] G. Antonelli, S. Chiaverini, N. Sarkar, and M. West. Adaptive control of an autonomous underwater vehicle: experimental results on odin. Control Systems Technology, IEEE Transactions on, 9(5):756-765, Sep 2001.

[3] E. A. Barros and F. J. A. Soares. Desenvolvimento de um robô submarino de baixo custo. In XIV Congresso Brasileiro de Automática, 2002.

[4] H. Bay, A. Ess, T. Tuytelaars, and L. V. Gool. Surf: Speeded up robust features. Computer. Vision and Image Understanding, 110(3):346-359, 2008.

[5] G. Benet, F. Blanes, J.E. Sim, and P. Prez. Using infrared sensors for distance measurement in mobile robots. Robotics and Autonomous Systems, 40(4):255 - 266, 2002.

[6] C. Benton, J. Kenney, R. Nitzel, D.R. Blidberg, S. Chappell, and S. Mupparapu. Autonomous undersea systems network (ausnet) - protocols to support ad-hoc auv communications. In Autonomous Underwater Vehicles, 2004 IEEE/OES, pages 83-87, June 2004.
[7] Mogens Blanke, Karl-Petter Lindegaard, and Thor I. Fossen. Dynamic model for thrust generation of marine propellers, 2000.

[8] J. H. R. Borenstein. Navigation Mobile Robots: Systems and Techniques. AK Peters, 1996.

[9] G. Conte and A Serrani. Modelling and simulation of underwater vehicles. In Computer-Aided Control System Design, 1996., Proceedings of the 1996 IEEE International Symposium on, pages 62-67, Sep 1996.

[10] J. P. V. S. Cunha. Projeto e estudo de simulação de um sistema de controle a estrutura variável de um veículo submarino de operação remota. Master's thesis, Federal University of Rio de Janeiro, 1992.

[11] R. B. Dominguez. Simulação e controle de um veículo submarino de operação remota. Master's thesis, Federal University of Rio de Janeiro, 1989.

[12] C. Benton. M. M. Haag, R. Nitzel, D. R. Blidberg, S. G. Chappell, and S. Mupparapu. Advancements within the ausnet protocol. In In Fourteenth International Symposium on Unmanned Untethered Submersible Technology, 2006.

[13] L. Hsu, J. P. V. S. Cunha, F. Lizarralde, and R. R. Costa. Avaliação experimental e simulação da dinâmica de um veículo submarino de operação remota. Controle \& Automação, 11(2):82-93, 2000.

[14] D.P. Miller. Design of a small, cheap uuv for under-ship inspection and salvage. In Autonomous Underwater Vehicle Technology, 1996. AUV '96., Proceedings of the 1996 Symposium on, pages 18-20, Jun 1996.

[15] S. Mupparapu, R. Bartos, and M. M. Haag. Performance evaluation of ad hoc protocols for underwater networks. In Fourteenth International Symposium on Unmanned Untethered Submersible Technology, 2005.

[16] P. Ridao, J. Batlle, and M. Carreras. Dynamic model of an underwater robotic vehicle. Technical report, University of Girona, 2001.

[17] F. Schweiger, B. Zeisl, P. Georgel, G. Schroth, E. Steinbach, and N. Navab. Maximum detector response markers for sift and surf. In Vision, Modeling and Visualization Workshop, 2009.

[18] E. Souza and N. Maruyama. An investigation of dynamic positioning strategies for unmanned underwater vehicles. In XIV Congresso Brasileiro de Automática, 2002.

[19] J. Yuh. Design and control of autonomous underwater robots: a survey. International Journal of Autonomous Robots, 1(3):1, 142000 . 